\title{
Consider species specialism when publishing datasets
}

To the Editor - The need to publish biodiversity data to ensure study replicability and to identify important conservation areas is an emerging challenge. Several journals now require publication of full data sets for published papers, and an increasing number of journals specialize in publishing only data sets. There is an evident trade-off between the undeniable importance of publishing fine-scale biodiversity data and the risk of several related threats ${ }^{1,2}$. Tulloch et al. ${ }^{1}$ highlight the importance of sharing biodiversity data and present a decision tree aiming to help researchers in making the decision of whether to publish their biodiversity data sets. This paper provides a much needed framework for researchers working on biodiversity, but, as for any pioneering work, we believe that consideration of additional aspects could allow more balanced and appropriate decisions.

For example, the decision of whether or not to publish specific data is particularly complex if species are ecologically or geographically restricted. We have been working for several years on the endangered European cave salamanders (genus Hydromantes) $^{3,4}$, which are a group of amphibians filling a narrow ecophysiological niche that can be found at high densities in subterranean environments characterized by specific microclimatic conditions $^{5-7}$. We tried to apply the decision tree $^{1}$ to the publication of Hydromantes data. Trade and exploitation are not the major threats to these animals; they are affected primarily by human disturbance, such as cave tourism and habitat alteration. No specific conservation policies exist to protect Hydromantes, and distribution data can be useful for their conservation. Therefore, we obtained the final suggestion: 'Restrict data: mask species IDs not locations or publish high-res habitat maps'.

For species that live in a particular habitat, as is the case of micro-endemisms, publishing detailed positions can instantly threaten the species even if species IDs are masked ${ }^{8}$. For example, if cave coordinates fall inside the range of one of the Hydromantes species, the location of the populations can easily be deduced. Likewise, when a species demands a particular combination of environmental features, it is not that hard to track its presence from a high-resolution habitat map $^{5,7}$. In this case, the publication of detailed sites and/ or high-resolution suitability maps will expose the species to immediate threats. The scenario is even more complex since data collection often requires collaboration with volunteers and funding agencies, which are reluctant to produce freely available data sets as this would overlook their financial and time inputs ${ }^{2,9}$. This may lead to a reduction of participation and of funding for conservation planning, which in turn results in a reduction of attention toward some endangered species ${ }^{9}$.

Publication of biodiversity data is a highly problematic topic, and an understanding of the underlying benefits and risks is just emerging ${ }^{1,2,9}$. We therefore recommend carefully evaluating the tradeoff between the effective protection of species and information sharing, even for conservation purposes. Particular attention should be paid to species with strong habitat specialization and narrow ranges.

Enrico Lunghi ${ }^{1,2 \star}$, Claudia Corti ${ }^{1}$, Raoul Manenti ${ }^{3}$ and Gentile Francesco Ficetola ${ }^{3,4}$

${ }^{1}$ Sezione di Zoologia "La Specola", Museo di Storia Naturale dell'Università degli Studi di Firenze, Firenze, Italy. ${ }^{2}$ Natural Oasis, Prato, Italy. ${ }^{3}$ Dipartimento di Scienze Politiche ed Ambientali, Università degli Studi di Milano, Milano, Italy. ${ }^{4}$ Laboratoire d'Écologie Alpine (LECA), CNRS, Université of Grenoble Alpes Grenoble, Gières, France. ${ }^{*}$-mail: enrico.arti@gmail.com

Published online: 11 February 2019 https://doi.org/10.1038/s41559-019-0803-8

References

1. Tulloch, A. I. T. et al. Nat. Ecol. Evol. 2, 1209-1217 (2018).

2. BenDor, T. K., Vitro, K. A. \& Riggsbee, J. A. Environ. Sci. Policy 74, 68-74 (2017).

3. Rondinini, C., Battistoni, A., Peronace, V. \& Teo li, C. Lista Rossa IUCN dei Vertebrati Italiani. (Comitato Italiano IUCN e Ministero dell'Ambiente e della Tutela del Territorio e del Mare, 2013).

4. Wake, D. B. Amphib-reptil. 34, 323-336 (2013).

5. Ficetola, G. F. et al. Sci. Rep. 8, 10575 (2018).

6. Lunghi, E. et al. J. Therm. Biol. 60, 79-85 (2016).

7. Lunghi, E. et al. Sci. Rep. 8, 7527 (2018).

8. Manenti, R. et al. J. Nat. Conserv. 45, 90-97 (2018).

9. Pearce-Higgins, J. W. et al. J. Appl. Ecol. 55, 2544-2551 (2018).

Competing interests

The authors declare no competing interests. 